\title{
NEW THERAPEUTIC OPTIONS IN UVEITIS
}

\author{
SUSAN LIGHTMAN \\ London
}

\begin{abstract}
SUMMARY
Most patients with sight-threatening posterior uveitis eventually end up on systemic medication to control their disease. Although the more aggressive approach to the use of these drugs does offer the patient a better chance of significant visual improvement at least in the short term, this is often associated with severe systemic side-effects in both the young and older patient. Cyclosporin has become a very useful second-line agent as a steroid sparer in those patients who can tolerate it. However, it is not suitable for or effective in everyone and the other currently available drugs are often of limited effectivity or associated with major systemic sequelae. This paper summarises the therapeutic approaches currently being examined to define whether they have a role in the better management of these patients in the future. Particularly exciting is the potential for sustained intraocular drug delivery so that adequate drug levels are achieved inside the eye without the necessity for systemic administration.
\end{abstract}

Uveitis remains a major cause of significant visual loss in the Western world. ${ }^{1}$ Sight-threatening complications such as cataract ${ }^{2}$ and glaucoma ${ }^{3}$ can now be managed much more effectively and safely in these patients but macular oedema, often causing profound loss of central vision, may or may not respond to conventional therapy. Posterior uveitis, which may or may not be associated with a systemic disease, is known to be a T-cell-mediated autoimmune disease. ${ }^{4}$ Current management consists of suppressing the immune system so as to dampen the inflammatory response, using either locally administered or systemic drugs. Corticosteroids remain the mainstay of treatment and can be given via the orbital floor or sub-Tenon's route in a depot preparation, ${ }^{5}$ or systemically either as an intravenous bolus or orally. In many patients, the inflammatory response is quietened and additional therapy is not required. Additional therapy is required when

Correspondence to: Professor Susan Lightman, Department of Clinical Ophthalmology, Moorfields Eye Hospital, City Road, London EC1V 2PD, UK. Tel: +44 (171) 566 2456. Fax: +44 (171) 251 9350. e-mail: s.lightman@ucl.ac.uk. steroids alone are not effective or too high a dose is required to achieve the desired effects. Second-line drugs given with the steroids in these situations include cyclosporin A, azathioprine and occasionally chlorambucil or cyclophosphamide.

Despite the use of high doses of these drugs, many patients will still lose vision from macular oedema or have serious systemic side-effects, which may preclude their further use, from the doses required to control their ocular disease. Other drugs which have immunosuppressive activity are now being used in patients with uveitis as well as the more commonly used drugs given via a different route. Surgical intervention and laser treatment have also been looked at again. New drugs and therapeutic approaches are being assessed in experimental models with interesting results.

\section{NEW DRUG THERAPIES IN CLINICAL USE Methotrexate}

Methotrexate (MTX) is now used as a second-line drug for rheumatoid arthritis and juvenile chronic arthritis in many centres but at a much lower dose than when used previously. Its exact mode of action at this low dose remains uncertain, but its antiproliferative effect is thought to be important. ${ }^{8,9}$ At low doses given in a one dose weekly pulse $(12.5 \mathrm{mg} /$ week or less), it is associated with few serious sideeffects. In the first ocular study, 12 patients with chronic non-infectious intraocular inflammatory disease, who had not responded to or who had become intolerant of corticosteroid or alternate cytotoxic agents, were treated weekly with oral low-dose pulse MTX and followed for up to 39 months. ${ }^{10}$ Response time ranged from 3 to 9 weeks after implementation of MTX therapy. Five of the 9 patients with chronic uveitis improved and 2 of the 3 patients with retinal vasculitis also had their inflammatory activity improved from moderate to mild. The others showed no improvement with the addition of MTX. It was found that MTX was more effective in treating patients with inflammatory pseudotumour, scleritis

Eye (1997) 11, 222-226 (C) 1997 Royal College of Ophthalmologists 
and orbital myositis than those with intraocular inflammatory activity.

In another study, 14 adult patients with active chronic non-infectious uveitis, who had failed to respond to previous systemic corticosteroid therapy were treated with low-dose MTX but with a different regime. ${ }^{11}$ MTX therapy was initiated in 8 patients at a dose of $40 \mathrm{mg}$ given intravenously once weekly for 4 weeks followed by $15 \mathrm{mg} /$ week given orally; 6 subjects were started on the oral regimen of $15 \mathrm{mg} /$ week. During a follow-up period of 3-24 months, intraocular inflammation improved in all subjects with MTX treatment and was associated with significant visual improvement in 11 .

Side-effects in both studies include mild elevation of transaminases, transient mucositis, partial alopecia and nausea. Clinically significant side-effects are rare and generally the drug has been well tolerated. Caution is required in patients with pre-existing hepatic or renal disease. Previous studies have shown it not to be very effective in the management of systemic vasculitis such as that associated with Wegener's granulomatosis or polyarteritis nodosa, ${ }^{12}$ and it is possible that it was more effective in intraocular inflammatory disease in the second study as a higher dose was used.

Thirty-two patients with endogenous uveitis resistant to treatment with high doses of cortisone and other immunosuppressive agents were treated with cyclosporin A (at an initial dose of $5 \mathrm{mg} / \mathrm{kg} / \mathrm{per}$ day, subsequently modified according to individual clinical response), fluocortolone and MTX. ${ }^{13}$ Disease remission was obtained in all patients, together with the recovery of normal visual acuity in 20 cases and its significant improvement in the remaining 12 . Furthermore, in all patients there was a significant decrease in all ocular inflammatory activity which was maintained for a period of 6-18 months. No signs of renal or hepatic toxicity were observed in any of the patients. A form of hirsutism appeared in only 2 cases.

MTX, perhaps in combination with steroids and cyclosporin at least initially, appears to have a role in the management of intraocular inflammatory disease and longer-term studies are now needed.

\section{Anti-CD4 $4^{+}$Monoclonal Antibodies}

It is known that $\mathrm{CD}^{+} \mathrm{T}$-cells are important in the perpetuation of posterior uveitis and anti-CD $4^{+}$ T-cell therapy using monoclonal antibodies has been used in the management of other autoimmune diseases known to involve $\mathrm{CD} 4^{+}$cells. A patient with long-standing refractory endogenous uveitis was treated with a chimeric monoclonal anti-CD4 antibody given intravenously. ${ }^{15}$ No immediate improvement was documented, but the frequency of uveitis relapses was sharply reduced after this therapy.
Interestingly, the patient's response to conventional therapy also improved after this treatment. Therapy with these chimeric antibodies is still limited due to the host antibody response engendered by them.

\section{FK506 (Tacrolimus)}

FK506 is in many ways a similar drug to cyclosporin and its use in patients has been limited to a few centres, particular in the transplantation field. Most of the published studies of its use in uveitis have come from Japan. In an open multicentre clinical trial to evaluate the efficacy and the adverse sideeffects of single therapy with FK506 in refractory uveitis 53 patients (41 patients with Behçet's disease, 5 with Vogt-Koyanagi-Harada (VKH) syndrome, 4 with idiopathic retinal vasculitis, and 3 with other forms of uveitis) were given FK506 in various doses for 12 weeks. ${ }^{16}$ Both the therapeutic effect and sideeffects were dose dependent. In $38 \%$ of patients treated with an initial dosage of $0.05 \mathrm{mg} / \mathrm{kg}$ per day, $60 \%$ at $0.10 \mathrm{mg} / \mathrm{kg}$ per day, $83 \%$ at $0.15 \mathrm{mg} / \mathrm{kg}$ per day and $79 \%$ at $0.20 \mathrm{mg} / \mathrm{kg}$ of body weight per day there was some improvement of disease control. The major side-effects were renal impairment $28.3 \%$, neurological symptoms $20.8 \%$, gastrointestinal symptoms $18.9 \%$, and hyperglycaemia $13.2 \%$. The trough level of FK506 in whole blood correlated with both the efficacy of the therapy and the incidence of adverse effects. On the basis of these results, a daily dosage of $0.10-0.15 \mathrm{mg} / \mathrm{kg}$ per day with the trough drug level between 15 and $25 \mathrm{ng} / \mathrm{ml}$ was suggested as an appropriate therapeutic dosage for refractory uveitis. A similar study also recommended the initial dose of FK506 to be $0.15 \mathrm{mg} / \mathrm{kg}$ per day based on efficacy and safety data, and confirmed that the trough drug level measured in whole blood should be between 15 and $25 \mathrm{ng} / \mathrm{ml}^{17}$

In another multicentre open clinical trial in Japan, 14 patients with non-infectious uveitis were given FK506 and the results reported. ${ }^{18}$ Seven patients had Behcet's disease, 5 had VKH syndrome, 1 had sympathetic ophthalmia and 1 idiopathic retinal vasculitis. Of the patients with Behcet's disease, 5 improved, 1 remained unchanged and 1 deteriorated. Of the patients with Vogt-Koyanagi-Harada syndrome, 4 improved and 1 remained unchanged. The patient with sympathetic ophthalmia improved but the patient with retinal vasculitis remained unchanged. Major adverse effects were as above and all adverse effects disappeared or improved when FK506 was stopped or when the dosage was decreased. Renal dysfunction and glucose intolerance appeared when the blood level of FK506 was high.

The major problem for us in interpreting these results for use in our patients is the difference in management approach between Japanese 
ophthalmologists and other centres dealing with certain uveitis problems. For example, systemic steroids are hardly ever used in patients with uveitis and Behçet's disease in Japan, so that a response to FK506 does not mean that the patients would not have responded equally well to conventional therapy such as steroids with or without cyclosporin. In patients with VKH syndrome, much larger doses of systemic steroids are given for much longer in Japan than in other centres, which may indicate disease of a much greater severity. This makes it difficult to evaluate the role for this drug in the management of patients in the UK. However, it is beginning to be used more in the transplantation field, particularly to see whether it is effective alone without the concomitant use of steroids or to allow a much lower dose of steroids to be used (steroid-sparing effect).

\section{NEWER DRUG THERAPIES UNDER EVALUATION}

\section{Intraocular Steroid}

Steroids are given to patients with uveitis by a variety of routes and intraocular steroid is often used peroperatively in patients undergoing vitrectomy for endophthalmitis. ${ }^{19}$ However, dexamethasone, the steroid currently used, has a very short half-life in the eye, particularly when the blood-ocular barriers are damaged. ${ }^{20}$ Many drugs can be given via the intraocular route (e.g. amikacin, vancomycin, amphotericin B, ganciclovir and foscarnet, but in chronic conditions longer-term intraocular drug delivery would be preferable. In the management of cytomegalovirus (CMV) retinitis in AIDS patients, repeated intravitreal injections of ganciclovir are often used very effectively, ${ }^{21}$ avoiding the systemic side-effects of the drug but allowing good control of the CMV retinal disease. Slow-release devices filled with ganciclovir ${ }^{22}$ are now available for insertion into the eye to avoid the necessity of repeated injections and can be safely exchanged several times when they no longer contain drug. ${ }^{23}$

Experimental studies in rabbits with induced uveitis suggest that slow-release devices containing dexamethasone are effective in treatment of severe panuveitis. On clinical examination, all signs of anterior and posterior uveitis were significantly less than in the untreated animals during the experimental time of 99 days, at which time the devices still contained a significant amount of drug. ${ }^{24}$ In addition the inflammatory response and tissue disorganisation as seen on histological examination was much less and late complications of the severe inflammatory process such as corneal neovascularisation, cataract and hypotony were also significantly reduced in the treated eyes. When the inflammation was re-induced, the treated animals again showed less active inflam- mation. After antigen rechallenge with the device in situ, inflammation in experimental eyes was still less than in controls.

In patients with exudative macular degeneration, a single dose of $4 \mathrm{mg}$ of intraocular triamcinolone, a longer-acting steroid, was given with the aim of flattening the posterior pole. ${ }^{25}$ This was successfully achieved without a significant rise in intraocular pressure or a greatly increased incidence of visually significant cataract. These two complications were only seen in patients given a further intraocular injection of triamcinolone. Slow-release devices containing dexamethasone are now in phase 1 clinical trials in the US in patients with refractory uveitis (Dr G. Jaffe, personal communication) and the outcome of these studies is eagerly awaited. If patients with localised ocular disease can be effectively managed entirely by administration of drugs given locally, this has to be a significant advance over their systemic use which, although effective, is often associated with severe systemic side-effects particularly in those patients of all age groups who require prolonged therapy.

\section{Induction of Immune Tolerance as Therapy}

Experimental studies have shown that feeding retinal proteins and peptides can induce a state of immune tolerance so that the animals are protected from disease. ${ }^{26}$ Mucosal administration of the retinal antigens can also induce this. ${ }^{27}$ In an open pilot study, patients fed large doses of retinal antigens were able to reduce their dose of immunosuppressive drugs or stop them altogether and remain in remission with good disease control on no therapy. ${ }^{28}$ This has also occurred in patients with multiple sclerosis who were fed myelin basic protein. ${ }^{29} \mathrm{~A}$ double-masked clinical trial is currently under way with patients who have intermediate uveitis on drug therapy and who are receiving either retinal antigens or placebo by mouth, and is due to report later this year (S. M. Whitcup, personal communication).

\section{EXPERIMENTAL STUDIES}

The animal model of experimental autoimmune uveoretinitis $(E A U)^{30,31}$ is widely used for the assessment of the effectiveness of drug therapy in uveitis. Many of the studies are on prevention of disease initiation, which is interesting but of less importance clinically than the treatment of established disease. Most recently mycophenolate mofetil,${ }^{32}$ rapamycin ${ }^{33}$ leflunomide, ${ }^{34}$ suramin, ${ }^{35}$ and the monoclonal antibodies anti-ICAM-1 and anti-LFA ${ }^{36}$ have all been tried successfully. It is hoped that clinical studies on some of these compounds will be carried out in the future when they have been further assessed in other fields of immunosuppression such as transplantation. 


\section{VITRECTOMY}

There have been various and conflicting reports in the literature over the years outlining the role of vitrectomy in the management of chronic posterior uveitis and in its subsequent clinical course. ${ }^{37}$ The main aim of this type of therapy is to reduce the need for long-term immunosuppressive therapy with all its systemic side-effects, to induce disease remission and to try to prevent the long-term sequelae of chronic intraocular inflammation such as macular oedema with its often devastating reduction of central vision. Patients with pars planitis and intermediate uveitis are often young, and for them anything which reduces the need for long-term systemic steroids is helpful. In a study of 25 eyes in patients aged between 5 and 18 years with intermediate uveitis and visual acuities between 0.02 and 0.4 (mean 0.19), vitrectomy was performed after failure of conservative treatment and peripheral cryotherapy. ${ }^{38}$ Twentytwo of the 25 eyes showed improvement in visual acuity within the first 6 months after surgery. Among the patients with a remarkable improvement in visual acuity, 7 eyes had chronic cystoid macular oedema which regressed post-operatively. No increase in lens opacities has been noticed, even after a follow-up period of 10 years.

In a further study, vitreous surgery was performed in 13 children (age range 2-15 years) with chronic uveitis and combined lensectomy-vitrectomy was performed in a further 6 eyes. ${ }^{39}$ Few post-operative complications occurred and 12 of the 19 eyes showed a significant visual improvement in the follow-up of between 6 months and 5 years. Preoperatively, 8 eyes had cystoid macular oedema, which showed regression in 7 of the oedema after surgery. In two-thirds of cases the intensity of the inflammation decreased, so that the dose of corticosteroids could be reduced post-operatively. This study differs markedly from that in which 9 patients aged from 63 to 86 years suffering from idiopathic multifocal chorioretinitis with panuveitis for which immunosuppressive drug therapy had no effect, underwent vitrectomy with no therapeutic benefit. ${ }^{40}$ Although visual improvement of one or two Snellen lines was achieved postoperatively, in most cases presumably from clearance of vitreous debris, the visual acuity decreased to preoperative values or less within 6 months.

\section{LASER TREATMENT}

Macula grid laser treatment has been shown to be beneficial in diabetic patients with non-ischaemic macular oedema and its use in chronic uveitic macular oedema has been assessed in a recent study. ${ }^{41}$ Six eyes of 5 patients with long-standing cystoid macular oedema due to chronic uveitis were treated by grid laser photocoagulation of the macula. A temporary increase in oedema with paracentral scotomas was observed in the first few weeks, but after more than 18 months the macular oedema had been reduced significantly or disappeared, as demonstrated by fluorescein angiography, in all treated eyes. One eye had a significant increase in Snellen acuity, three eyes more or less stabilised, and two eyes deteriorated. The beneficial effect of laser treatment on visual acuity in patients with uveitis might be greater if performed at an earlier stage of the disease.

Key words: Uveitis, Immunosuppression, Steroids, Macular oedema, EAU, Vitrectomy.

\section{REFERENCES}

1. Rothova A, Suttorp-von Schulten MS, Frits Trefers WF, Kijlstra A. Causes and frequency of blindness in patients with intraocular inflammatory disease. $\mathrm{Br} \mathrm{J}$ Ophthalmol 1996;80:332-6.

2. Barton K, Hall AJH, Rosen P, Cooling RJ, Lightman S. Systemic steroid prophylaxis for cataract surgery in patients with posterior uveitis. Ocular Immunol Inflammation 1994;2:207-16.

3. Towler HNA, Bates AK, Broadway DC, Lightman S. Trabeculectomy with 5-fluorouracil for glaucoma secondary to uveitis. Ocular Immunol Inflammation 1995;3:163-70.

4. Lightman S, Chan C. Immune mechanisms in chorioretinal inflammation in man. Eye 1990;4:345-53.

5. Riordan-Eva P, Lightman S. Orbital floor steroid injections in the treatment of posterior uveitis. Eye 1994:8:66-70.

6. Lightman S. Use of steroids and immunosuppressive drugs in the management of uveitis. Lancet 1991;338: $1501-4$

7. Rosenbaum JT. Treatment of severe refractory uveitis with intravenous cyclophosphamide. J Rheumatol 1994;21:123-5.

8. Olsen NJ, Murray LM. Antiproliferative effects of methotrexate on peripheral blood mononuclear cells. Arthritis Rheum 1989;32:378-85.

9. Calabrese LH, Taylor JV, Wilke WS, et al. Response of immunoregulatory lymphocyte subsets to methotrexate in rheumatoid arthritis. Cleve Clin J Med 1990; 57:232-41.

10. Holz FG, Krastel H, Breitbart A, Schwarz-Eywill M, Pezzutto A, Volcker HE. Lose-dose methotrexate treatment in non-infectious uveitis resistant to corticosteroids. Ger Ophthalmol 1992;1:142-4.

11. Shah SS, Lowder CY, Schmitt MA, Wilke WS Kosmorsky GS, Meilser DM. Low-dose methotrexate therapy for ocular inflammatory disease. Ophthalmology 1992;99:1419-23.

12. Wilke WS, Biro JA, Segal AM. Methotrexate in the treatment of arthritis and connective tissue diseases. Cleve Clin J Med 1987;54:327-38.

13. Pascalis L, Pia G, Aresu G, Frongia T, Barca L. Combined cyclosporin A-steroid-MTX treatment in endogenous non-infectious uveitis. J Autoimmun 1993; 6:467-80.

14. Hahn HJ, Kuttler B, Laube F, Emmrich F. Anti-CD4 therapy in recent-onset IDDM. Diabetes Metab Rev 1993;9:323-8.

15. Thurau SR, Wildner G, Reiter C, Riethmuller G, Lund OE. Treatment of endogenous uveitis with anti-CD4 monoclonal antibody: first report. Ger J Ophthalmol 1994:3:409-13. 
16. Mochizuki M, Masuda K, Sakane T, Ito K, Kogure M, Sugion N, et al. A clinical trial of FK506 in refractory uveitis. Am J Ophthalmol 1993;115:763-9.

17. Sakane T, Mochizuki M, Inaba G, Masuda K. A phase II study of FK506 (tacrolimus) on refractory uveitis associated with Behçet's disease and allied conditions [in Japanese]. Ryumachi 1995;35:802-13.

18. Isioka M, Ohno S, Nakamura S, Isobe $K$, Watanabe $N$, Ishigatsubo Y, Tanaka S. FK506 treatment of noninfectious uveitis. Am J Ophthalmol 1994;118:723-9.

19. Schulman JA, Peyman EA. Intravitreal corticosteroids as an adjunct in the treatment of bacterial and fungal endophthalmitis: a review. Retina 1992;12:336-40.

20. Stern GA. Factors affecting the efficacy of antibiotics in the treatment of experimental post-operative endophthalmis. Trans Am Ophthalmol Soc 1993;91: 775-844.

21. Young SH, Morlet N, Heery St, et al. High dose intravitreal ganciclovir in the treatment of cytomegalovirus retinitis. Med J Aust 1992;157:370-3.

22. Anand R, Nightingale SD, Fish RH, et al. Control of cytomegalovirus retinitis using sustained release of intraocular ganciclovir. Arch Ophthalmol 1993;111: 223-7.

23. Morley M, Duker J, Ashton P, Robinson M. Replacing ganciclovir implants. Ophthalmology 1995;102:388-92.

24. Cheng CK, Berger AS, Pearson PA, Ashton P, Jaffee GJ. Intravitreal sustained-release dexamethasone device in the treatment of experimental uveitis. Invest Ophthalmol Vis Sci 1995;36:442-53.

25. Penfold P, Gyory J, Hunyor A, Billson F. Exudative macular degeneration and intravitreal traimcinolone: a pilot study. Aus NZ J Ophthalmol 1995;23:293-8.

26. Nussenblatt RB, Caspi R, Mahdi C, Cahn C, Roberge $\mathrm{F}$, Lider $\mathrm{O}$, Weiner HL. Inhibition of S-antigen induced experimental autoimmune uveoretinitis by oral induction of tolerance with S-antigen. J Immunol 1990;144:1689-95.

27. Dick AD, Cheng YF, Liversidge J, Forrester JV. Intranasal administration of retinal antigens suppresses retinal antigen-induced experimental autoimmune uveoretinitis. Immunology 1994;82:625-31.

28. Nussenblatt RB, Whitcup SM, de Smet MD, Caspi RR, Kozhich AT, Weiner HL, Vistica B, Gery I. Intraocular inflammatory disease (uveitis) and the use of oral tolerance: a status report. Ann N Y Acad Sci 1996; 778:325-37.

29. Hohol MJ, Khoury SJ, Cook SL, Orav EJ, Hafler DA,
Weiner HL. Three-year open protocol continuation study of oral tolerization with myelin antigens in multiple sclerosis and design of a phase III pivotal trial. Ann NY Acad Sci 1996;778:243-50.

30. Faure JP. Autoimmunity and the retina. Curr Top Eye Res 1980;2:215-302.

31. Caspi RR, Roberge FG, McAllister CG, El-Saied M, Kuwabara T, Gery I, Hanna E, Nussenblatt RB. T-cell lines mediating experimental autoimmune uveoretinitis (EAU) in the rat. J Immunol 1986;136:928-33.

32. Chanaud NP III, Vistica BP, Eugui E, Nussenblatt RB, Allision AC, Gery I. Inhibition of experimental autoimmune uveoretinitis by mycophenolate mofetil, an inhibitor of purine metabolism. Exp Eye Res 1995; 61:429-34.

33. Roberge FG, Xu D, Chan CC, de Smet MD, Nussenblatt RB, Chen $\mathrm{H}$. Treatment of autoimmune uveoretinitis in the rat with rapamycin, an inhibitor of lymphocyte growth factor signal transduction. Curr Eye Res 1993;12:197-203.

34. Robertson SM, Lang LS. Leflunomide: inhibition of Santigen induced autoimmune uveitis in Lewis rats. Agents Actions 1994;42:167-72.

35. Sartani G, Silver PB, Strassmann G, Chan CC, Caspi RR. Suramin treatment suppresses induction of experimental autoimmune uveoretinitis (EAU) in rodents. Curr Eye Res 1995;14:887-96.

36. Uchio E, Kijima M, Tanaka S, Ohno S. Suppression of experimental uveitis with monoclonal antibodies to ICAM-I and LFA-I. Invest Ophthalmol Vis Sci 1994; 35:2626-31.

37. Verbraeken $H$. Therapeutic pars planitis vitrectomy for chronic uveitis: a retrospective study of the longterm results. Graefes Arch Clin Exp Ophthalmol 1996; 234/5:288-93.

38. Kroll P, Romstock F, Grenzebach UH, Wiegant W. Early vitrectomy in endogenous juvenile uveitis intermedia: a long-term study [in German]. Klin Monatsbl Augenheilkd 1995;206:246-9.

39. Bacskulin A, Eckardt C. Results of pars plana vitrectomy in chronic uveitis in childhood. Ophthalmologe 1993;90:434-9.

40. Nolle B, Eckardt C. Vitrectomy in multifocal chorioretinitis. Ger J Ophthalmol 1993;2:14-9.

41. Suttorp-Schulten MS, Feron E, Postema F, Kijlstra A, Rothova A. Macular grid laser photocoagulation in uveitis. Br J Ophthalmol 1995;79:821-4. 